\title{
Concerning the Coefficient of Storage of Aquifer (2)
}

\section{Gunjiro SAKAI*}

\begin{abstract}
In the last paper "Concerning the Coefficient of Storage" publised in this jouthal Vol.14 $(1968)^{(')}$, the writer advocated a thinking way of the decreasing of ground-water storage by pumpage and, as the result, diminishing of coefficient of storage, which was a kind of measure to evaluate the ground-water storage. But he described also in the paper that his advocation was no more than an idea and did not reach its conclusion.

Through the study thereafter, the writer noticed an inperfection in the thinking way or the treatment of the unit of pumpage period $t$. Then he corrected them and discovered an easy graphycal solution, under the condition remaining transmissibility concerned unchangeable.
\end{abstract}

\section{Treatment of Transmissibility $T$}

The argument of the writer is grounded on the opinion of Robinson and Skibizke(1962)(.). They advocated a conception as "Maximum Possible Drawdown" by pumpage and denoted it by the letter $s_{\text {max }}$. Their argument stands upon the Theis' non-equillibrium formula and by the procedure to differetiate the drawdown, that is, the drop by pumpage $s$ by the transmissibility $T$ is developed. For the convenience of argument, the writer must describe again the three equations derived by them.

$$
\begin{aligned}
& s_{\max }=(0.647) \frac{Q}{4 \pi T} \cdot \cdots \\
& T=(0.575) \cdot r^{2} \cdot \frac{S}{t} \cdot \cdots \\
& s_{\max }=\frac{(0.281) Q}{r^{2}} \cdot \frac{t}{S}
\end{aligned}
$$

here $S$ is the coefficient of storage, $t$ is the time elapsed after pumping start of withdrawal $Q$, and $r$ is the distance of observation well from the pumping well, where $s$ or $s_{\max }$ is observed.

The withdrawal $Q$ is prescribed by the drop $s$ and the transmissibility $T$ of the aquifer concerned. Though Robinson and Skibizke differentiated $s$ by $T$, it is not nescessary to consider that $T$ is variable. The writer described also in the last paper as "it is reasonable to think that the variability of $T$ may be only apparent due to the reflection of $S$ ". But it is proper to think that $T$ is unvariable and maintained constant always by the two variable factors $S$ and $t$ contained in Equ. (2). Recently, regarding with the phenomena as earth subsidence, the variation of $T$ has been discussed. But from the point of well hydraulics, $T$ has been treated as a constant value for concerning aquifer, and it is not 
any exception for the derivation of the Theis' non-equillibrium formula.

\section{The Character of $s_{\max }$}

As Equ. (3) expresses, $s_{\max }$ must change according to the two variable factors $S$ and $t$, even when the withdrawal $Q$ is maintained constant. Although $s_{\max }$ seems to be constant by Equ. (1), the argument originally stands upon the non-equillibrium theory, such a situation never occurs. Therefore, Equ. (1) must be applied to a certain time section of pumping duration, that is, $s_{\max }$ must change according to $t$ in general.

If $s_{\max }$ is differentiated by $t$, the next equation would be derived from Equ. (3).

$$
\begin{gathered}
\frac{\partial s_{\max }}{\partial t}=K \cdot \frac{1}{S}\left(1-\frac{t}{S} \cdot \frac{d S}{d t}\right) \\
K=\frac{(0.281) Q}{r^{2}}
\end{gathered}
$$

At a certain time section when the maximum possible drawdown $s_{\max }$ is maintained as a constant practically, $\partial s_{m a x} / \partial t$ must become zero, then the next equation is derived.

$$
1-\frac{t}{S} \cdot \frac{d S}{d t}=0
$$

By the transforming and integrating Equ. (5), ne can obtain the next equation.

$$
\log _{e} t=\log _{e} S+C
$$

here $C$ is a certain integral constant.

Hereupon, if we assume $S_{o}$ and $t_{o}$ as the optional original values of $S$ and $t$, and introduce the conditions as $S_{o}>S$ and $t_{o}<t$, then the next equation would be derived from Equ. (6).

$$
\begin{aligned}
& \log _{e} t_{o}=\log _{e} S_{o}+C \\
& \log \frac{t}{t_{o}}=\log \frac{S_{o}}{S}=\log \frac{1}{S / S_{o}}
\end{aligned}
$$

\section{Graphycal Evaluation of Coefficient of Storage}

If we assume $s_{\max \cdot 0}$ as the optional original value of $s_{\max }$ corresponding to $t_{o}$ and $S_{o}$, the next equation may be derived from Equ. (3).

$$
\frac{s_{\max }}{s_{\max \cdot 0}}=\frac{t / t_{o}}{S / S_{o}}
$$

By trnsforming in logarismic form and substituting Equ. (7), the next two equations are obtained.

$$
\begin{aligned}
& \log _{e} \frac{s_{\max }}{s_{\max \cdot 0}}=\log _{e} \frac{t}{t_{0}}+\log _{e} \frac{S_{o}}{S} \\
& \log \frac{s_{\max }}{s_{\max \cdot 0}}=2 \log \frac{t}{t_{o}}
\end{aligned}
$$

As the inserted figure snows, if the relation curves expressing Equ. (7) and Equ. (9) are ploted in'a logarismic section paper, $s_{\max } / s_{\max \cdot 0}$ and $S / S_{o}$ in the ordinate and $t / t_{o}$ in the abscessa, two straight lines would be drawn, the one whose gradient is 2 shows the relation between $s_{\max } / s_{\max \cdot 0}$ and $t / \overrightarrow{t_{0}}$ (Line I), and the another whose gradient is 1 shows 
teh relation $S / S_{0}$ and $t / t_{0}$ (Line II).

The procedure for the practical use of the relation curves as follows:

1) From an optional value ef $s_{\max } / s_{\max \cdot 0}$, a line parallel to the abscessa is drawn, then the value of $t / t_{0}$ is known by the corresponding intersection with Line I.

2) A line parallel to the ordinate which connects the intersection mentioned above and the corresponding $t / t_{0}$ intersects with Line II. The value of $S / S_{o}$ corresponds to the intersection is the being required one.

In this graphycal procedure, the unit of time and the time of pumping start are out of question, because all values concerned on the curve figure are indicated in the form of ratio and dimensionless. It seems very curious that this graphycal procedure does not concern with either withdrawal $Q$ or. transmissibility $T$. But as Equ. (1) shows, $s_{\max }$ is already regulated by the two factors mentioned above. In practical, $s_{m a x}$ is measured and then $s_{\max }$ is assumed. Therefore, the regulation by $Q$ and $T$ is reflected in the value of $s_{m a x}$. . Concerning this problem, however, some studies may be necessary in future.

This figure expresse that $t / t_{o}$ and $S / S_{o}$ are 2.0 and 0.25 respectively, when $s_{\max } / s_{\max \cdot 0}$ is 6 . The situation means that if the time elapsed from pumping start $t_{0}$ is five years and $s_{\max \cdot 0}$ is $10 \mathrm{~m}$, the ratio of the coefficient of storage would diminish to 0.25 after 20 years, i.e. at the time $t 30$ years, for an assumed $s_{\max } 60 \mathrm{~m}$.

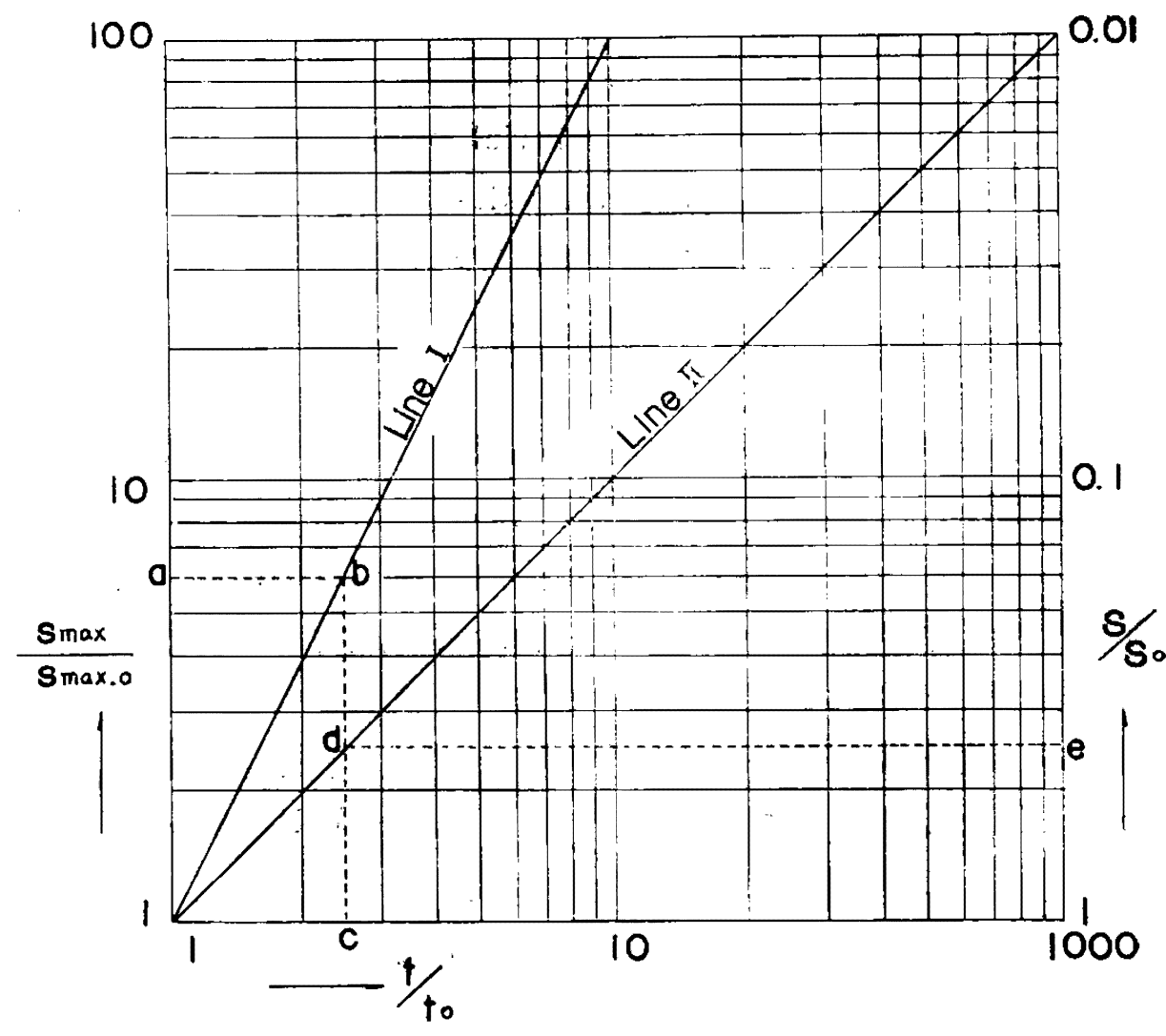

References

(1) Sakai,G.: Concerning the Coefficient of Storage of Aquifer, Journal of Japanese Association of Groundwater Hydrology, no.14, 1968, July.

(2) Robinson, G. M., and H. E. Skibizke: A Formula for Computing Transmissibility Causing Maximum Possible drawdown due to Pumping, U. S. G. S., Water-Supply Paper 15.3:-F, 1962. 